\title{
Students' evolving meaning about tangent line with the mediation of a Dynamic Geometry environment and an Instructional Example Space
}

\author{
Irene Biza \\ Loughborough University \\ Address: Mathematics Education Centre \\ Loughborough University \\ Loughborough LE11 3TU \\ UK \\ Telephone number: $\quad$ +44(0)150922 2877 \\ Fax number: $\quad$ +44 (0)1509228211 \\ e-mail address: $\quad$ i.biza@,lboro.ac.uk
}

\begin{abstract}
In this paper I report a lengthy episode from a teaching experiment in which fifteen Year 12 Greek students negotiated their definitions of tangent line to a function graph. The experiment was designed for the purpose of introducing students to the notion of derivative and to the general case of tangent to a function graph. Its design was based on previous research results on students' perspectives on tangency, especially in their transition from Geometry to Analysis. In this experiment an instructional example space of functions was used in an electronic environment utilising Dynamic Geometry software with Function Grapher tools. Following the Vygotskian approach according to which students' knowledge develops in specific social and cultural contexts, students' construction of the meaning of tangent line was observed in the classroom throughout the experiment. The analysis of the classroom data collected during the experiment focused on the evolution of students' personal meanings about tangent line of function graph in relation to: the electronic environment; the pre-prepared as well as spontaneous examples; students' engagement in classroom discussion; and, the role of researcher as a teacher. The analysis indicated that the evolution of students' meanings towards a more sophisticated understanding of tangency was not linear. Also it was interrelated with the evolution of the meaning they had about the inscriptions in the electronic environment; the instructional example space; the classroom discussion; and, the role of the teacher.
\end{abstract}

Keywords: Tangent Line; Mediation of Meaning; Instructional Example Space; Dynamic Geometry Software; Local Straightness 


\section{Introduction}

Functions and related concepts in Analysis (e.g. limit, derivative, integral etc.) have a wide field of applications in mathematics and other disciplines. Introductory courses in Analysis constitute a basic part of the mathematical curriculum of secondary education and in many countries good student performance in these courses is necessary for university entry. Furthermore, as several studies show, many students are challenged by the above concepts not only during their secondary studies but, even-more, at post secondary level (for a review see Artigue, Batanero and Kent 2007; Harel, Selden and Selden 2006). These challenges have been addressed by innovative didactical approaches some of which utilise electronic environments to enhance students' understanding (for a review on use of technology in Calculus see Tall, Smith and Piez 2008).

The work discussed in this paper is the third and final stage of a study of Year 12 and first year university students' learning and understanding of tangent line to a function graph. These students had met the concept of tangent in different mathematical contexts (Euclidean and Analytic Geometry and Analysis) and in several representational systems (figural, graphical, symbolic, verbal). The objectives of this study were: to investigate the students' perspectives on tangents built in the course of their transition between mathematical and representational contexts; and, to explore ways in which the tangent line could be introduced while taking these perspectives into consideration. With regard to the first objective, the first two stages of the study proposed a model describing what perspectives on the relationship between a curve and its tangent are created by students in their transition from the Euclidean Geometry to Analysis (Biza 2008; Biza, Christou and Zachariades 2008; Biza and Zachariades 2010). With regard to the second objective, in the third stage of the study, on which this paper is based, a teaching experiment was designed, carried out and evaluated in order to introduce Year 12 students to the notion of derivative and the general case of tangent to a function graph.

The results of the first two stages of the study formed the basis for the design of the teaching experiment in which a Dynamic Geometry (DG) environment was utilised. In this experiment the researcher became a teacher with the purpose to investigate firsthand students' mathematical learning and reasoning (Steffe and Thompson 2000) and to gain better insight into students' constructions of mathematical meaning. This paper reports three conjectures that this experiment aimed to examine. The first conjecture is that students' personal meaning of a definition - in this case the definition of tangent line of function graph varies from the mathematical meaning even if they had been introduced to this definition and had been exposed to an electronic environment rich in representations. The second one is that this personal meaning can evolve significantly in a specific teaching and learning context. Finally, the third one is that this evolution occurs in interaction with the evolution of the teaching and learning context. 
The teaching experiment had the characteristics of the diagnostic teaching methodology proposed by Bell (1993) that

"consists of identifying the key conceptual points and common misconceptions within the topic, using existing research, and testing directly the students who are to be taught. The teaching is then designed to focus on these points, giving the students substantial challenges, provoking cognitive conflict by exposing misconceptions, and resolving them through discussion" (ibid, p. 115).

In designing the experiment attention was given to the potentially significant role of the DG environment, to the examples and to the type of formal mathematical symbolism to be used during the teaching experiment. In this paper I report a lengthy episode from the teaching experiment in which the classroom negotiates the definition of tangent line to a function graph. The analysis focuses on the evolution of the students' personal meaning of the definition in relation to: the mediation of the inscriptions of the mathematical concepts in the DG environment and the examples used; students' engagement in classroom discussion; and, the role of the researcher as teacher.

To this end, firstly, I present the theoretical background of the study consisting of the research results and the theoretical constructs on which the study was based. The research results regard the teaching and learning of Analysis with a focus on students' understanding of tangent line and the role of technology. These results informed the diagnostic teaching methodology of the study regarding the necessary, according to Bell (1993), conceptual points and common misconceptions. The theoretical constructs, on the other hand, frame the study's focus regarding students' formation of mathematical meaning; the semiotic mediation of tools; and, the importance of discussion and the role of examples in students' construction of meaning. Then, I introduce the method of the study and I present and analyse the episode (in seven interlinked incidents). Finally, I conclude with a discussion on the role of the DG environment, the examples, the classroom discussion and the researcher as a teacher in students' construction of meaning of the tangent line.

\section{Theoretical background}

\section{Students' difficulties with concepts of Analysis: The case of the tangent line}

Research has highlighted the relation between students' perspectives on tangents and their views on functions and function graphs in general. The concept of function and its properties can be regarded from different points of view. For instance, according to a global point of view a function and its properties are considered within its domain whereas through a local point of view - a point of view that characterises Analysis - attention focuses on what happens at a specific value and in its neighbourhood. Maschietto (2008) claimed that the transition from the global to the local point of view and vice versa ( $\mathrm{global} /$ local game) is important for an introduction to Analysis. Students' misinterpretation of tangents has been ascribed to their difficulties to deal with this transition (e.g. Biza et al. 2008; Castela 1995). For example, 
in Biza et al. (2008) students who had met the concept of tangent in different mathematical contexts (Euclidean and Analytic Geometry, Analysis) demonstrated several Intermediate perspectives on tangents between the Analytical Local perspective - the tangent is defined through the slope or the derivative applied locally at the tangency point - and the Geometrical Global perspective - the tangent preserves geometrical properties applied globally on the entire curve. These Intermediate perspectives are a fusion of geometrical and analytical properties applied either globally on the entire graph or locally in a neighbourhood of the graph. For example, students with these perspectives often believe that the tangent line: could have more than one common point with the curve under the condition that there is only one common point in a neighbourhood of the tangency point or should have only one common point with the curve regardless of whether it splits or not the curve at that point (Biza et al. 2008). These perspectives of tangency drive students towards erroneous or contradictory responses in cases where the tangent line has more than one common point or when it coincides with the curve - as well as in cases of inflection, edge and cusp points ${ }^{1}$.

The above difficulties that students face in their encounter with tangents also relate to difficulties they often have with the generalisation of the definition of a concept, particularly when they have already encountered specific cases of the same concept. This generalisation requires the reconsideration of all previously met specific cases in the light of the general definition. Hence the role of the defining properties is important as they assist classification of all the cases encountered so far under the same general term. On the contrary, learners often regard a property recurring in some of the cases of a concept as a general defining statement for all the cases (Winicki and Leikin 2000). For example, students' early experiences of the circle tangent contribute to their creation of an image of the tangent as a line that touches the graph at one point only and does not cross it (Tall 1987; Vinner 1991).

\section{Using technology for teaching Analysis: The case of tangent line}

Students' understanding of notions of Analysis has been connected with the ways that these notions are represented (e.g. Presmeg 2006). For example, for the purpose of deeper conceptual understanding in Analysis, instruction should be focused not only on the use of algebraic representations but additionally should take into account the geometric and intuitive representations of the corresponding mathematical objects as well as the interactions among these multiple representations (Kaput 1994). Such modes of instruction have been gaining strength also due to the advances in computer technology and the development of dynamic mathematical software (Habre and Abboud 2006).

\footnotetext{
${ }^{1}$ We call inflection point of a function graph, a point in which the concavity of the curve changes and there is a tangent line at this point; edge point a point in which the function is continuous and the derivative from the left and the right exist without being equal; and, cusp point a point in which the function is continuous and the limit of the rate of change from the left and the right is infinite.
} 
Several approaches have been introduced for teaching concepts in Analysis (e.g. limit, derivative, integral etc.) with technological means (for a review see: Tall et al. 2008). The approach adopted in this experiment built on the dynamic/visual idea of local straightness (Tall 1989) or micro-straightness (Maschietto 2008). This idea refers to the fact that, if we focus close enough to a point of a function graph, a point in which the function is differentiable, then this graph looks like a straight line. This 'straight line' is the tangent line of the graph at this point. The local straightness not only characterises a graph of a differentiable function but also offers a way of understanding, for example, nondifferentiability of a function (Tall et al. 2008): absence of local straightness suggests nondifferentiability at a particular point. This approach is often supported by zooming tools in appropriately designed software (Tall 1989) or in a graphic calculator (Maschietto 2008) and facilitates student engagement with the local/global game that is so important for a transition to Analysis (Maschietto 2008).

\section{Theoretical constructs that frame the study}

According to the Vygotskian (1978) approach knowledge develops in ways that are influenced by the specific social and cultural context. This point of view suggests that there is a strong relationship between the individuals and the social world around them according to which the meanings are shaped under the mediation of the culture in the form of tools and in particular of language. These tools are psychological tools or signs such as words, graphs, mathematical symbols, or physical tools. The construct of semiotic mediation is introduced by Vygotsky (1978) to describe the process in which an intermediate link (e.g. a sign) mediates between the stimulus and the response. In the Vygotskian sense, applied in the contemporary educational context, the inscriptions (graphs, symbols etc.) of electronic environments can be thought as signs of mathematical theory and as such may become mediators in students' interaction with this theory (Marriotti 2001). This mediation expands students' conceptions of mathematical objects (symbols, shapes etc.), especially towards generalisation and formalisation (Noss and Hoyles 1996). The notion of mediation has proved useful for analysing a range of interactions between the user and the environment, including interactions sometimes unforeseen by teachers and designers (Lagrange 1999). Furthermore, within electronic environments, a teacher can find tools which can mediate in a mathematical discussion in ways that facilitate students' transition from strictly personal to more mathematical meanings (Mariotti 2001).

At this point I would like to clarify the distinction between students' personal meaning and mathematical meaning with the latter corresponding to what is acceptable and used by the mathematical community. In the school classroom the more knowledgeable is the teacher and he/she acts as the carrier of this mathematical meaning. When students encounter a new mathematical sign, this sign may not have any meaning for them. Later, they start to use the sign through the interaction with the teacher within the zone of proximal development (ZPD, Vygotsky 1978). At the beginning their understanding may be unstable 
and immature but their personal meaning evolves through communication and functional use of the sign (Berger 2004). Bartolini Bussi (1996) raised the role of this communication as form of mathematical discussion that is:

"the scientific debate that is introduced and orchestrated by the teacher on a common mathematical object in order to achieve a shared conclusion about the object that is debated upon (e.g. a solution of a problem). In this case the teacher utters a voice that represents the mathematical culture: the perspective on the object that is introduced by the teacher is usually different from the ones that are introduced by the pupils." (ibid, p. 17)

In addition, the role of the discussion has proved essential in students' introduction to a new mathematical definition. Asking students to construct, consider and evaluate definitions in a group discussion has the potential to bring to the fore students' insights into their personal constructions of specific mathematical concepts as well as their understandings about the nature and role of a mathematical definition (Zaslavsky and Shir 2005).

Furthermore, this discussion can be richer and more meaningful to the students if it is based on specific examples. Learners' participation in a discussion of examples and counterexamples often offers them the opportunity to encounter conflict and the resolution of this conflict may lead to modification of their knowledge (Lakatos 1976; Watson and Mason 2005; Zaslavsky and Shir 2005). The role of examples is crucial in the introduction of new concepts (Watson and Shipman 2008) and in the development of students' ability to generalise (Zazkis, Liljerdah and Chernoff 2008). An attentive choice of examples can help students make appropriate generalisations or refute inappropriate generalisations. But caution is needed as not every set of examples will lead to successful generalisations (Zazkis et al. 2008). Watson and Mason (2005) in their discussion of example spaces - namely collections of examples that fulfil a specific function - define conventional example spaces "as generally understood by mathematicians and as displayed in textbooks" (ibid, p.76). This may differ from the students' example spaces, which can be either personal and based locally on their own past experiences or collective and situated locally to a classroom or other group at a particular time (ibid). Zazkis and Leikin (2008) refined the notion of conventional example space and made the distinction between "“expert' example spaces, that display rich variety of expert knowledge and 'instructional' example spaces, as displayed in textbooks and most often used in instruction" (ibid, p. 132). The role of the teacher is important in the use of the instructional example space "not only to seek appropriate examples, but to develop specialising strategies among learners" (Zazkis et al. 2008, p. 141).

In this study instructional example spaces are considered as collections of examples used by teachers and students as they work on a particular notion in an instructional context. These examples are prepared by the teacher in advance and may be amended under the influence of students' responses in the course of the instruction. In this sense the instructional example space draws on the teacher's example space, is shaped by his/her mathematical and pedagogical knowledge and may change during the instruction. 
The study presented in this paper focuses on the evolution of students' personal meaning of tangents in a classroom discussion through the mediation of the inscription of local straightness in the DG environment and the instructional example space prepared especially for the needs of the teaching experiment. The analysis considers the essential role in the experiment of the researcher as teacher.

\section{Methods}

\section{The students}

The participants of this study were 15 Year 12 students (aged 17-18 years) of a Greek secondary school who had taken mathematics as a major subject. At the time of the experiment, the students had been taught functions in general and limit and continuity of a function and they were about to be introduced to the derivative and its applications. In their previous studies, they had encountered the tangent line to the circle in Euclidean Geometry (Year 10) and to other conic sections (parabola, hyperbola and ellipse) in Analytical Geometry (Year 11) courses. The students had used Dynamic Geometry environments in their previous Euclidean Geometry courses.

\section{The classroom setting}

The teaching experiment took place over two sessions, the first lasting for one hour and the second two hours. The experiment was run by the researcher (myself) with the support of the classroom teacher. The teacher was experienced in teaching mathematics with IT and taught Geometry to this same group of students with Dynamic Geometry environments.

For the needs of the experiment, the mathematics classroom was transformed into a laboratory with five portable computers and a sixth linked to an overhead projector. The students worked in groups of three in each of the five computers and they kept notes on worksheets prepared for the needs of the experiment. During the experiment I was operating the sixth computer connected with the projector, keeping notes on the blackboard and wandering around the class in order to monitor the progress of group work and orchestrate the classroom discussion.

\section{Data collection}

Data was collected through pre- and post- teaching experiment questionnaires, audio recording of the whole-class discussion during the experiment, students' responses on the worksheets and my notes. A narrative of the experiment was produced (field text, Clandinin and Connelly 2000) consisting of field notes, transcripts and commentary. For the purposes of this paper I present details and analysis of a combination of the above data with a focus on one lengthy episode which I have divided into seven incidents. The analysis of the episode focused on the evolution of the students' personal meaning of the definition in relation to: their use and interpretation of the inscriptions in the DG environment,; their 
participation in the discussion; the examples I offered the students; and, my choices - regarding the examples and the orchestration of the discussion - as a teacher.

\section{The didactical aims of the experiment}

The design of the experiment was based on the research results, presented earlier, about students' understanding of tangents. The aim of the experiment was the reconstruction of previous, restricted perspectives about tangents and the creation, through the introduction of derivative, of a more general understanding about tangency - including an understanding of tangent at inflection points, tangent that meets the graph at another point or coincides the graph as well as cases in which the tangent cannot be defined (e.g. at an edge point). To this end the experiment intended to deploy the dynamic visual graphics in the electronic environment (e.g. the magnified image) and the symbolic expressions (e.g. the limit of rate of change) as mediators in students' formation of the meaning of tangent line. In accordance with these aims, the teaching experiment had the following stages:

1. Examination of generalisable properties of tangency (e.g. the tangent line as the limiting position of secant lines and the linear approximation of the curve) in the case of circle.

2. Examination of the above properties in the case of semicircle.

3. Introduction to the definition of the derivative and the tangent to a function graph.

4. Establishment of these definitions in classroom discussion through critical examples of function.

\section{The electronic environment}

The electronic environment used in the experiment was developed in the framework of a European Commission funded project called CalGeo for "Teaching Calculus with Dynamic Geometry Tools" (Biza, Diakoumopoulos and Souyoul 2007). At the time that the project was conducted, many studies of the teaching and learning of derivative and tangent line had deployed Function Grapher and Analyser as the appropriate electronic environments for the introduction of these notions and their properties (Maschietto 2008; Tall 1989). For this study the Dynamic Geometry software called EucliDraw (http://www.euclidraw.com), that combines Dynamic Geometry (DG) and Function Grapher (FG) facilities, was employed. This choice was driven by the aims of the CalGeo project and the specific experiment. According to these aims the electronic environment should facilitate students in:

- Their transition from the geometrical context to the analytical context and the connection between these contexts.

- Their exploration of mathematical properties across different but connected representations (e.g. graphical and symbolic).

- Their access to different mathematical aspects of the same concept, in this case the tangent line.

To meet the above aims we needed an electronic environment that:

- Offers a friendly interface that integrates geometrical (DG) and analytical (FG) properties. 
- Offers different and connected representations.

- Facilitates the easy access to accurate, or as accurate as possible in an electronic environment, inscriptions of different aspect of the same notion and in different example.

I will describe briefly the electronic environment used in the experiment and how it is considered appropriate to satisfy the above requirements. In EucliDraw both DG and FG facilities are available at the same time offering an environment appropriate for integrated application in which the DG capabilities are smoothly linked with the FG capabilities. Indicatively, I refer to the magnification tool that can magnify a region of any point on the screen in a separate window (Figure 1). This magnification can be repeated as many times as the user specifies through a magnification factor and the graph and its magnification are presented at the same time on the screen. Technically, the highly magnified graph is produced by recalculation and redesign of the graph. As a result, the magnified graph is graphically reliable, the curve is not deformed and the image is not restricted by the size of the pixels (about these technical issues see Tall et al. 2008).

Especially for the needs of the experiment, a specific electronic environment was designed (Figure 1) with the following constructions: a function graph (in Figure 1, $y=\sin (x)$ ); a movable point $A\left(x_{0}, f\left(x_{0}\right)\right)$ on the function graph; a parameter $h$ and the related points $B\left(x_{0}+h, f\left(x_{0}+h\right)\right)$ and $C\left(x_{0}-h, f\left(x_{0}-h\right)\right)$; the secant lines $A B$ and $A C$ and their slopes; and, the magnification window of a region of $A$ related to a magnification factor equal to $1 / h$. The user can change the position of point $A$ and the values of $h$ causing the dynamic changes of the other related items (e.g. points $B$ and $C$ secant lines, slopes etc.). When $h$ decreases (approaches zero), points $B$ and $C$ move closer to $A$; the secant lines and their slopes change; and the magnification factor and the zooming-in capacity increase, accordingly. The function formula and the corresponding graph can be changed without any effect on other parts of the construction (e.g. $h$, secant lines, magnification window etc.).

[Insert Figure 1 about here]

This environment implements and extends the design principles offered by previous research (Maschietto 2008; Tall 1989) by offering simultaneously access to the global view (image of the entire graph) and the local view (image of a magnified part). Moreover, in this approach, the local straightness is connected explicitly with the tangent line and both of them with the limiting position of secant lines and their limit. Also, the opportunity of the easy construction of geometrical shapes (e.g. circles or parallelograms) proved to be crucial in the experiment (Biza, 2010). Finally, the fact that the function graph can be changed easily without any effect on the other parts of the construction offers the opportunity for the easy alternation between different examples of functions. Given that the discussion of several examples is crucial in the design of the teaching experiment, this facility proved important. 


\section{The instructional example space}

This experiment took into account results from previous studies regarding students' understanding on tangent line (Biza et al. 2008; Castela 1995; Tall 1987; Vinner 1991). According to these studies students build a personal meaning about tangent that may be inappropriate for cases of tangency as the tangent to an inflection, edge and cusp point or in cases in which the tangent line has more than one common point with the graph or coincides with the graph. The examples, that appear in school textbooks and are used in teaching practice in the Greek educational context in which the study was conducted, do not consider the entire range of the above examples. This may result in restricted understanding of the notion of tangent line (Biza and Zachariades 2010). In this experiment the instructional example space (Zazkis and Leikin 2008) was expanded in order to include a richer series of examples that aimed to support the construction of a rich meaning of tangent line of function graph. Some of these examples were counterexamples to the inappropriate properties students attribute to the tangent line. They aimed to create conflict encouraging students to rethink whether their perspectives about tangent line are correct or not.

\section{Student responses to the pre-questionnaire}

In the pre-questionnaire - distributed 20 days before the teaching experiment - the 15 students were invited to: describe curves for which they supposed that there is a tangent line; give an informal definition of this line and some of its properties; and, construct or to identify the tangent line of given curves at a point $A$. These curves and the corresponding point $A$ were selected in order to cover a variation of cases of tangency that research has identified as problematic in students' understanding about tangency such as cases in which point $A$ is an inflection, edge or cusp point or cases in which the tangent line has more than one common point with the graph or coincides with the graph (Biza et al. 2008; Castela 1995; Tall 1987; Vinner 1991). Some indicative examples of the curves on which the students were asked to construct or to identify the tangent lines as well as the number of correct responses are presented in the first two lines of Table 1.

In the identification and construction tasks, students' performance varied depending on the curve and the position of the point $A$. Generally students performed well in cases in which there is a tangent line that has only the tangency point in common with the curve and leaves the curve in the same semi-plane. They also performed well in cases in which the curve is a conic section (ellipse, hyperbola or parabola). The students' performance worsened in the tasks in which the tangent line and the curve have more than one common point - especially when the tangent line or part of it coincides with the curve (see the first five graphs in Table 1). More than half of the students answered correctly in the cases in which the point $A$ is an edge point (see the last graph in Table 1). However, when the point A is an inflection point, although more than half of the students identified the tangent correctly, only a few of them managed to construct the correct tangent line (see the $6^{\text {th }}$ and the $7^{\text {th }}$ graph in Table 1 ). 
Concerning the definition of tangent line and its properties, eleven students recalled properties from the tangent to a circle such as: "they have one common point", "the line keeps the figure in the same semiplane" and "the tangent is vertical to the radius". One of these students wrote that the line kept the curve at the same semi-plane "locally" - I return to this student's hint at the locality later in the postquestionnaire responses. Some students made a not necessarily relevant reference to derivative ( 7 students) or to algebraic terms and processes (15 students) such as: "slope of the line"; "the equation of the line"; "system of two equations"; and, conics formulae.

[Insert Table 1 about here]

\section{An episode on the students' evolving meaning about tangent line}

The episode in this section relates to students' negotiation of the definition of tangent line to a function graph. I selected this episode in order to illustrate what meanings students had created about tangent line after their first introduction to the definition and how these meanings evolved through the mediation of the electronic environment and the use of the specific examples. As the episode is rather long I will present the critical points of this evolution. I will describe briefly what had preceded the episode and then I will go through seven critical incidents that marked the shifts in the students' meaning of the definition.

\section{Preceding the episode.}

The episode took place just after the students' introduction to the tangent line of a function as the limiting position of secant lines, the definition of derivative and the construction of the slope of tangent and its formula through the derivative at the corresponding point. I will briefly describe what occurred in the previous stages of the experiment in order to give the context of the episode that follows. In the first stage of the experiment, the tangent to a circle was discussed. Also, the property of local straightness at a point $A$ in relation with the limiting position of the line $A B$, as the point $B$ was approaching point $A$, was investigated in the electronic environment. Then the curve was changed to a semicircle (stage 2) and after that to the graph of $\sin x$ (stage 3). In stage 3, the image of the graph in the magnification window (Figure 1) in relation to the limiting position and the slope of the secant lines $A B$ and $A C$ - as $h$ approached zero was considered. Through this investigation, the following became apparent: the secant lines approached the same position (coincided), the slopes of the two lines converged to the same value and the function graph became straight in the magnification window. In this activity some students conjectured that the line to which the secants converged could be the tangent line of the function graph at the point $A$ and they noticed that this line had slope equal to the limit of the slopes of the secant lines. Through this conjecture, and with the use of the symbolic language of limits, the definition of the tangent line of function graph at 
a point $A\left(x_{0}, f\left(x_{0}\right)\right)$ in the context of Analysis was introduced as: a line with formula $y-f\left(x_{0}\right)=f^{\prime}\left(x_{0}\right)\left(x-x_{0}\right)$ and slope equal to $\lim _{h \rightarrow 0} \frac{f\left(x_{0}+h\right)-f\left(x_{0}\right)}{h}$, defined as the derivative of the function at $x_{0}\left(f^{\prime}\left(x_{0}\right)\right)$.

According to the lesson plan (stage 4) the above definitions of derivative and tangent line were to be established in classroom discussion through critical examples of functions. However, although students had been introduced to these definitions, their personal meaning about tangency had not yet become apparent. In order to trigger these students' meanings, I asked them: "what do you believe is the definition of the tangent line of a function graph at a point $A$ ?" with the expectation of responses related to what we had discussed earlier (e.g. based on limiting processes, derivative, etc.).

In the rest of the section I take the reader through the episode that occurred after this question. During this episode, students proposed definitions, I kept records of these definitions on the blackboard and I orchestrated the discussion using specifically prepared examples in the electronic environment. The proposed definitions emerged and transformed gradually through the classroom discussion (Table 2). These changes of definitions mark the seven incidents I track in the following presentation. In each one of the incidents I describe what happened in the classroom followed with my reflective comments. If deemed necessary, I introduce the reader to some contextual information at the beginning of the incident.

\section{Incident 1: Uncovering students' meaning about tangent line}

The first definition proposed by a student was that "the tangent is a line that has only one common point" and I wrote on the blackboard: the tangent line of a function graph is a line that has only one common point with the graph (Def. 1 in Table 2). When I questioned the students as to whether they approved the suggested definition, another student responded: "tangent is a line for which there is a point [whose coordinates] satisfy it [the equation of the line] and the function formula". He then added "the solution of the system of the line and function's equations is that point”. In accordance with his suggestion I wrote on the blackboard: the tangent line of a function graph is a line for which there is a point that satisfies it and the function formula (Def. 2 in Table 2). Soon after, a third student proposed: "the slope of the line equals the slope of the function graph". When I asked him what he meant by "slope of the function graph" he responded: "the derivative of the function". I asked him for his definition and he proposed the following, which I also wrote on the blackboard: the tangent line of a function graph is a line that has the same slope with the graph (its slope is equal to the derivative of the function) at a certain point (Def. 3 in Table 2).

The first definition is based in a geometrical argument related to the number of common points between the line and the graph as the characteristic property of tangency. In the second definition, the student used words like equations and system of equations adopting a more algebraic approach. In the third definition analytical terms such as slope, derivative and equality of slopes are used. In the three proposed definitions 
there are elements derived from the three mathematical contexts in which the students had met tangents before (geometrical, algebraic and analytical). However, there is no explicit connection between each of the three proposed definitions and the corresponding mathematical contexts. For example, the second definition hints at the common point(s) without any mention of the number or uniqueness of these point(s). Perhaps it was proposed by the student in order to suggest appropriate algebraic support for Def. 1. Furthermore, the third definition could be regarded as a contribution towards the completion of the second one. We could say that there is polyphony in the students' expressed views; also that there is no explicitly shared meaning about tangent line between the students, and the students and myself as their teacher. In addition, there was no reference to the local straightness, the secants and the limits in these spontaneous proposed definitions even though we used them in the prior part of the lesson. I considered the three proposed definitions as a challenging basis from which to initiate a mathematical classroom discussion towards the construction of a shared meaning of the definition of tangency.

\section{Incident 2: Students' meaning about tangent line shifts from global to local perspectives}

In order to elicit students' reflection on the first definition I moved point $A$ in the projected construction to a position in which the limiting position of secant lines cut the graph at another point (Figure 2a). I then invited the students to comment on the image. The students stated that the tangent line could have other common points - apart from the tangency one - and asked me to erase the first definition from the blackboard. In addition to this, a student proposed: "So, we can say that it [the tangent line] has one common point in a neighbourhood of point $A$ " and asked to change the definition as follows: the tangent line of a function graph is a line that has only one common point with the graph in a neighbourhood of the tangency point (Def. 4 in Table 2). As I mentioned to the students, we would have the opportunity to discuss this definition later in our session.

During the above recourse to the projected image it became apparent that the students were ready to accept the projected image in the electronic environment as perceptual evidence of tangency. The image in Figure 2a evoked the spontaneous acceptance on the part of the students of the projected line as tangent that had another common point without the need for more justification. However, it was not evident that the magnified image supported this acceptance. It could be the image of the entire curve and the line that convinced the students. At least, the magnified image was not in conflict with the students' arguments. In addition, it appears that the student who proposed Def. 4 assimilated the new image of tangency to the previous one by applying the property of one common point locally in a neighbourhood of the tangency point. This reaction agrees with previous research results about Intermediate perspectives on tangency in which geometrical properties are applied in a region of the tangency point (see Intermediate Local perspective in Biza et al. 2008). The student's definition is based mainly in the geometrical property of the one common point and is still using geometrical arguments as in Def.1. The difference is that, through the mediation of the projected image in the electronic environment, the meaning of the existence of 
common point has shifted from a global view (it concerns the entire graph) to a local view (it concerns a region of the tangency point).

\section{Incident 3: Students' arguments turn Analytical}

For me as teacher, the next issue for discussion, related to the second definition, was whether the existence of one or at least one common point was sufficient condition for tangency. To this aim, I chose to demonstrate, in the projected construction, a line which had one common point with the graph but did not have the same slope with it at this point (Figure $2 \mathrm{~b}$ ). This led the classroom to reject the second definition and made the student who had proposed the third definition (Def. 3 in Table 2) claim that "they have to have the same slope". I then asked the students whether "having the same slope only is a sufficient condition for tangency". To this aim I chose to sketch in the projected construction a line that was parallel with the limiting positions of secant lines without having any common point with the graph (Figure 2c). That made the same student propose the following modification and combination of the second and third definition that I wrote on the blackboard: the tangent line of a function graph is a line that has (at least) one common point with the graph and it has the same slope with the graph (its slope is equal to the derivative of the function) at the tangency point (Def. 5 in Table 2).

In this incident the aim was to discuss further the issues of existence of common points and the equality of slopes as sufficient or not conditions for tangency (Def. 2 and Def. 3 in Table 2) and construct a more appropriate definition by combining both properties. The selected examples illustrated the necessity of the modification of both conditions and the creation of the fifth definition through their fusion. The students' definitions seemed to turn gradually from geometrical properties (number of common points) into analytical properties (slope and derivative). They used the appropriate terms (slope, derivative) and processes (equality of slopes, existence of common point) correctly. However this evidence of analytical thinking does not guarantee that the students had constructed a sufficient meaning on tangency and that their understanding shifted permanently towards becoming analytical - as the next incidents reveal. Also we do not have clear evidence on the role of the magnified image in students' construction of meaning. The magnified image could mediate this construction or it could exist as a meaningless demonstration without any special use for the students.

[Insert Figures 2 about here]

\section{Incident 4: Lack of local straightness gives meaning to the magnified image}

According to the initial lesson plan, some examples of tangency had to be discussed: non-differentiable functions (edge point); tangency at an inflection point; tangent vertical to the $x x$ ' axis; and tangent that coincides with the curve. Some of the examples used so far resulted as reaction to the students' proposed 
definitions. At this point, I decided to proceed to the examples in my lesson plan in parallel with - and in support of - the ongoing discussion of the definition of tangent line. So far, the fourth and the fifth definitions (Table 2) were still on the blackboard.

I invited the students to change the formula of the function presented in their computers into $f(x)=|\sin (x)|$, move the point $A$ to the origin $(0,0)$ and observe the image of the graph, the secant lines and their slopes as $h$ approached zero. The students observed the non-convergence of $A B$ and $A C$ 's slopes and the image of the curve making an edge in the magnification window (Figure 3). I then asked them to express the partial limits of the slopes symbolically (namely $h$ tends to 0 from positive and negative values). The students observed that the partial limits are unequal and that the derivative cannot be defined. When I asked them to consider this observation in tandem with Def. 5 which was still on the blackboard, they proposed adding a condition to the fifth definition, as follows: the tangent line of a function graph at a point $A$ - if the function is differentiable at this point - is a line that has a common point with the graph at point $A$ and it has the same slope with the graph at this point (Def. 6 in Table 2). Through the $|\sin x|$ example we had the opportunity to discuss cases in which the function is nondifferentiable at a point and thus the tangent at this point does not exist. We tried to connect the nondifferentability of the function, visualized by the lack of local straightness, with the non-convergence of the rate of change. It was the first time in this episode that we recalled the symbolic expression for limits used in earlier stages of the experiment (stage 3). The students carried out this transition smoothly, demonstrating skills developed in their previous school experience in symbolic manipulation of limits. The aim was that the students would achieve a more sophisticated insight into local straightness through considering a case where local straightness was lacking. Sometimes students believe that the curve looks straight in the electronic environment for non-mathematical reasons (e.g. because of the screen resolution) (Biza 2010). If this is the case, the image provided by the electronic environment does not mediate in students' thinking about tangency. The connection between the local straightness and the mathematical properties of the curve can be emphasised through cases in which the curve does not look straight regardless of the level of magnification (ibid). Until this incident, there was no clear evidence of the meaning local straightness had for the students, and if this meaning was connected with the existence or not of tangent line. It is to the credit of the particular electronic environment and the specific example of a non-differentiable function that the students started to build a meaning of local straightness that connected the figural and symbolic inscriptions. We can say that the lack of local straightness in the magnified image supported students towards giving a meaning to this image. In addition, there was some evidence that this meaning started to be connected with the existence, or not, of tangent line.

[Insert Figure 3 about here] 


\section{Incident 5: The magnified image in conflict with students' meaning about tangent line}

In the next step, following the lesson plan, I invited the students to change the function in their computers to $f(x)=x^{3}$, move the point $A$ to the origin and observe the image of the graph, the secant lines and their slope, as $h$ approached zero. In this case the function is differentiable at the point $(0,0)$, the lines $A B$ and $A C$ and their slopes converge, the image of the curve looks like a straight line in the magnification window and the tangent line exists and splits the graph in two parts (Figure 4). I then asked the students if the graph had a tangent at point $(0,0)$ and a student responded: "it looks like [a tangent] but on the other hand it cannot be". When I asked him "why can it not be?" he said:

It cannot be a tangent because it cuts the graph ... how can I say it ... it splits it ... I mean it divides it in two parts, the one is on the one side and the other is on the other ... on the other hand it looks like it coincides near the $A$ in the magnification ... I don't know ...

I then asked "why does it bother us that the line cuts the graph?" and another student responded: "that is ... could it cut it ... split it?". When I replied "why not? Did we add any such restriction in the definition we have given?", a third student wondered: "that is, the properties we know from the circle are not valid in this case?".

[Insert Figure 4 about here]

Previous research has shown that students struggle with the image of tangency at an inflection point as they expect that a tangent keeps the curve at the same side at least in a neighbourhood of a tangency point (Biza et al. 2008; Castela 1995; Tall 1987; Vinner 1991). Informed and prepared by these results, I recalled the above example even though this perception was not explicitly present in the definitions proposed by the students thus far. In spite of the previous discussion, the students' perspectives on tangency appeared to remain related to geometrical properties, namely the remaining of the graph in the same side of the line. Although evidence of analytical thinking had emerged in the previous incidents of the episode, the example of the tangency at an inflection point caused a regression to students' earlier, mostly geometrical arguments. Even so, the image of local straightness in the magnification window had started to bring about some uncertainty in the ways students saw tangency. I consider this shift crucial. The mediation of the magnified image resulted in the students' conflict between their under-developed meaning about tangents and their previously established geometrical understanding, which was largely based on the relative positions of the line and the curve. At this point we can claim that local straightness had a meaning for the students which was related to the existence or not of tangent line. However, the students' previous perspectives based on circle were still strong. As a result, their arguments were swaying between the image of the circle tangent and the image in the magnification window, trying to settle down to an image that the students would find definitive and persuasive. 


\section{Incident 6: The magnified image mediates the extension of students' meaning about tangent line (but not for all students)}

I then invited the students to change the formula of the function in their computers into $f(x)=\sqrt{|x|}$, move the point $A$ to the origin and observe the image of the graph, the secant lines and their slope, as $h$ approached zero. The point $(0,0)$ in this case is a cusp point. That is, the function is not differentiable at the point $(0,0)$ but the graph looks straight in the magnification window, the secants converge to the same line and, although the limits of their slopes are infinite, we could say that the tangent does exist and it is vertical to the $x$-axis (Figure 5a).

The students were working on changing the values of $h$ and observing the image in the magnification window. In parallel, some of them were engaged in the calculation of the partial limits of $A B$ and $A C$ 's slopes (when $h$ tended to 0 from positive and negative values). In response to my question "is there a tangent line at $(0,0)$ ?", a student mentioned the magnified image and the results in his calculations of limits and concluded that there is a tangent line and proposed: "it [the tangent] doesn't have a slope because it is vertical to $x^{\prime} x$ and thus we could say that its slope is infinite"

In the discussion that followed another student added: "The point $O$ is a corner point". At that point I returned to my computer and I projected the magnified (around $O$ ) graphs of $f(x)=\sqrt{|x|}$ and $f(x)=|\sin (x)|$ (Figures 5a and 5b, respectively). In Figure 5b (see also Incident 4) the curve maintains the edge point in the magnification window regardless of the level of zooming. The graph in Figure 5a, on the other hand, has a linear approximation and looks straight. These two images, projected in parallel and in a way that allowed their comparison, made the student above doubt her previous comment and say: “Ah, they are not the same things ...". But she couldn't express what their difference was.

A bit later in this discussion, I asked the students to adjust Def. 6 in order to include the case of the vertical to the $x x^{\prime}$ axis line. Following some deliberations with the students, I wrote on the blackboard:

The tangent line of a function graph at a point $A\left(x_{0}, f\left(x_{0}\right)\right)$ is the line with equation $y-f\left(x_{0}\right)=f^{\prime}\left(x_{0}\right)\left(x-x_{0}\right)$, if the function is differentiable at $A$

or

the line with equation $x=x_{0}$, if $f$ is continuous at $x_{0}$ and the limits $\lim _{h \rightarrow 0^{+}} \frac{f\left(x_{0}+h\right)-f\left(x_{0}\right)}{h}$ and $\lim _{h \rightarrow 0^{-}} \frac{f\left(x_{0}+h\right)-f\left(x_{0}\right)}{h}$ are infinite (Def. 7 in Table 2).

[Insert Figure 5 (5a and 5b) about here] 
A student then commented that, in all the cases we had discussed, "the tangent is the limiting position of the secant lines $A B$ and $A C$ " and I added the following definition on the blackboard:

The tangent line of a function graph at a point $A\left(x_{0}, f\left(x_{0}\right)\right)$ is the limiting position - if it exists of the secant lines $A B$ and $A C$ for $B\left(x_{0}+h, f\left(x_{0}+h\right)\right)$ and $C\left(x_{0}-h, f\left(x_{0}-h\right)\right)$ as $h$ approaches zero (Def. 8 in Table 2).

In addition to the above, another student said: "the tangent seems to coincide with the graph in the magnification window and when this does not happen then we don't have a tangent line".

Student difficulties with cusp points have been identified in previous research (Vinner 1991). I expected students' reluctance to accept the existence of the tangent at this point. However, drawing on the magnified image, the student above built an argument that the tangent line exists and is vertical to the $x$ axis; and, connected this argument with the results he had obtained from his calculation of the relevant limits. I could say that, for this student, the local straight image in the electronic inscription had the meaning of the existence of tangent; and, that this meaning was so clear that it mediated towards extending this student's definition in order to include the case of the vertical to the $x x$ ' axis tangent. The image - in the magnification window - of the curve looking straight and vertical to the $x x$ ' axis was connected with the infinite limits of slopes and led him to the conclusion that there is a tangent with 'no', or 'infinite', slope.

On the other hand, the student who called $O$ a "corner point" regarded the two graphs $(f(x)=\sqrt{|x|}$ and $f(x)=|\sin (x)|)$ as similar and considered only some, superficial, perceptual characteristics of the two graphs in her justification. In Greek secondary school mathematics jargon the term corner point is used for edge points. For example, the point $O(0,0)$ is an edge point for $f(x)=|\sin (x)|$ (see Incident 4) but not for the function under discussion (here $f(x)=\sqrt{|x|}$ ). This was the reason for which I decided to project the images of both graphs in the magnification window (Figure 5a and 5b). For this student the image in the magnification window did not have the meaning of the existence of tangent and she used the term corner point, that she might have heard in another context, disconnected from the magnified image. To me, her argument was in conflict with what was discussed in the classroom at this particular moment. But, to her, her argument was probably valid, as her personal meaning about the magnified image was not the same as mine or as to that of some of her peers.

Her comment however gave a great opportunity to compare the two graphs in the magnification window in connection with the symbolic representation of the limit (of the rate of change) - an opportunity that had not been included in the initial lesson plan. The selected example of the function $f(x)=\sqrt{|x|}$ and the spontaneous intervention that led to the comparison of the two graphs had significant impact on the lesson: they revealed differences in the students' interpretations of the figures in question; they 
strengthened the persuasive power of the magnified image; and, they resulted in a rather sophisticated discussion about tangents.

Finally the last comment about the coincidence of the graph and the line in the magnification window indicated that the student was forming a new understanding for the evaluation of whether a line is a tangent or not. I proposed to the students that they keep this student's suggestion in mind, also in connection with Def. 7 and 8, as it would prove handy for solving tangent line related mathematical problems.

\section{Incident 7: The magnified image supports the shift of students' meaning about tangent line}

Until this point, the fourth definition was still on the blackboard as none of the cases we had discussed so far was appropriate to unsettle its approval by the students. In order to trigger a discussion on this definition I invited students to change the function formula into: $f(x)=x^{2}-5$ for $x \leq 0$ and $f(x)=-5$ for $x>0$. In this case the tangent line at the point $(0,-5)$ does not satisfy the fourth definition as it coincides with the graph for all non-negative values (Figure 6).

[Insert Figure 6 about here]

Following work on this example, students suggested the rejection of the fourth definition. The student who had recommended this definition, earlier in Incident 2, then mentioned: "So, finally, none of the properties we knew from the circle is valid". Grasping the opportunity offered by this comment, I invited one more time the students to consider the circle as a special case of curve and reflect on its tangent through the limiting position of the secant lines and the image in the magnification window as we had discussed earlier.

Students' difficulties in accepting as a tangent a line that coincides with the graph at any region of the tangency point have been highlighted in previous research (Biza et al. 2008; Castela 1995; Tall 1987; Vinner 1991). With the support of this example we tackled another argument, related to the common points between the curve and its tangent, commonly characterising the students' hitherto mostly geometrical arguments. The student's spontaneous agreement with the rejection of all the properties known from the circle was an indication that at least this student was considering the possibility of a meaning shift about tangency. It seems that now the unstable meanings observed in the previous incidents were settling down as more solid analytical arguments, more and more distant from their initial geometrical characteristics. 


\section{Evolution of students' meaning of the local straightness and the definition of tangent line}

In the incidents presented above, I tracked a lengthy episode of classroom discussion on the definition of tangent line of function graph. In these incidents the students and I negotiated a series of relevant, partially relevant or irrelevant definitions proposed by the students and listed on the blackboard in the order presented in Table 2 .

[Insert Table 2 about here]

These definitions emerged in the classroom discussion through the mediation of the electronic environment and through specific examples of function graphs. In this process, not only students' meaning about tangent line evolved but the meaning students have been giving to the magnified image evolved. In order to describe this evolution across the episode, in Figure 7, I connected the examples under discussion (second line) with the definition transformations (third line) and the students' meaning of local straightness as it was evident across the incidents (fourth line). During the episode, the students' argument about tangency moved back and forth between geometrical (e.g. number of common points, relative position of line and graph) and analytical properties (e.g. equality of slopes, derivative) of tangent line. The dynamic graphs in the electronic environment (e.g. secant lines, image in the magnification window), the dynamic numeric values (e.g. values of $h$, secants' slope) and the symbolic expression (e.g. limits, rate of change) are not evident in the students' arguments at the very beginning of the discussion (Incident 1). Then, through the use of specific examples, the dynamic graphs, gradually, started to support students towards elaborating their perspectives about tangency. This support facilitated the shift of the students' meaning from global to local perspectives (Incident 2) and the emergence of analytical views (Incident 3). But, even in these incidents, the role of the magnified image in the evolution of meaning was not evident. It might act as a mediator but this mediation was not apparent in the students' arguments. Later, the magnified image, through the lack of local straightness, and in combination with the symbolic expressions, started to gain some meaning in relation to the tangent line (Incident 4). This meaning became apparent when it caused conflict when the students' views appeared to regress to a geometrical perspective (Incident 5). Also, this meaning of the magnified image - at least for some students mediated the extension of the definition of tangent line when this line was vertical to the $x$-axis (Incident 6) and supported the students' meaning shift from geometrical perspectives to analytical (Incident 7). The role of the examples was crucial in the above evolution. In these incidents, the examples prompted the discussion and fed constantly into, and were fed by, the evolution of the students' meaning (see, for example, Incident 6).

[Insert Figure 7 about here] 


\section{Student responses to the post-questionnaire}

The post-questionnaire contained analogous tasks to those in the pre-questionnaire. Some indicative examples of the tasks, the number of correct responses in the pre- and the post-questionnaire and the differences between the two tests are presented in Table 1. In the identification and construction tasks of tangent line the students performed better after the experiment. However, the tasks in which the tangent coincided with the curve or the tangency point was an inflection point were still the most challenging tasks for the students (Table 1).

Additionally, students' justifications in the questionnaire changed slightly. Although some responses were still under the influence of geometrical properties, some others were clearly enriched by the issues discussed in the experiment. For example, a student had responded in the pre-questionnaire that:

The tangent of a curve is a line that has only one common point 'locally' with the curve called tangency point. [...]. In addition if the curve 'locally' splits the plane in two semi-planes, the tangent belongs to only one of them. [student's quotation marks]

This student followed this definition consistently through all his responses in the pre-questionnaire. However, in a task in which he had to sketch a tangent at an inflection point, he wrote: "I believe that there is a tangent. But this case refutes the definition I gave earlier and I cannot express another one". The same student in the post-questionnaire wrote:

[a tangent] is the limiting position of a random secant line when the two common points with $C_{f}$ [the graph of the function $f]$ tend to coincide and take the coordinates $\left(x_{0}, f\left(x_{0}\right)\right.$. In addition, the tangent is

a 'local' notion in a sense that we do not bother about what happens in relation with the curve and the tangent 'far away' from the point under consideration [student's quotation marks]

This student had a local perspective on tangency even before the experiment. However, for this student the tangent line had geometrical meaning and he could not cope with subtle cases of tangency (e.g. at an inflection point). After the experiment the meaning changed and no geometrical properties were used any more.

\section{Conclusions}

In this paper I presented an episode from a Year 12 teaching experiment that focused on the introduction to the tangent line of a function graph, and its relationship to the derivative. The teaching experiment had the characteristics of the diagnostic teaching methodology proposed by Bell (1993). According to this approach, firstly, key conceptual points and misconceptions regarding tangent line of function graph should be regarded through reference to existing research and by testing directly the students to be taught. Then the teaching is designed to focus on the above points, giving the students challenges, provoking cognitive conflict by exposing potential misconceptions, and resolving them through discussion. 
According to the above, the experiment was based on research results on students' perspectives on the tangent line - especially influenced by their previous experience on the notion (e.g. the tangent to a circle) - that may not allow them to generalise it in the case of a function graph (Biza et al. 2008; Biza and Zachariades 2010; Castela 1995; Tall 1987; Vinner 1991). According to these results, students' perspectives about tangents were expected to be strongly influenced by the mathematical contexts and the representational systems in which they had met the notion before. Students who participated in this study demonstrated evidence of this influence in their responses to the pre-questionnaire.

Previous research has proposed that approaches that use electronic environments with zooming tools can facilitate students' engagement with analytical concepts (Tall et al. 2008). One of these approaches adopts the dynamic / visual idea of local straightness (or micro-straightness) (Maschietto 2008; Tall 1989). This experiment used the idea of local straightness that was developed in a Dynamic Geometry with Function Grapher facilities environment and aimed to contribute to our understanding of the influence of these environments on student understanding by examining the following three conjectures: students' personal meaning of the definition of tangent line of function graph varies from the mathematical meaning, even if students had been introduced to this definition and had been exposed to an electronic environment rich in representations (local straightness included); students' personal meaning of the definition can evolve significantly in a specific teaching and learning context; and, this evolution occurs in interaction with the evolution of the teaching and learning context. This learning context has four main characteristics: the electronic environment, and especially the inscription of local straightness in the magnified image; the pre-prepared, as well as spontaneous, examples that constitute the instructional example space; students' engagement in a classroom discussion; and, the role of researcher as a teacher. Following the Vygotskian (1978) approach according to which students' knowledge develops in ways that are influenced by the specific social and cultural context, I regard students' construction of the meaning of tangent line to occur in the classroom throughout the experiment. In this concluding part of the paper I will offer a more elaborate discussion on students' meaning of the definition of tangent line and its evolution during the experiment in relation to the above four characteristics.

At the beginning of the episode, and later on, there was evidence that the first conjecture was correct. Students, before the episode, had been introduced to the definition of derivative and the tangent line; had worked in their computers with some cases of curves; had observed the secant lines approaching each other; and, had seen the magnified image of the graph in a region of the tangency point. However, when they were asked to offer the definition of tangent line, they proposed several spontaneous definitions with no reference to the local straightness, the secants and the limits (see incident 1). Even later, there were cases in which students regressed to definitions of tangent that were irrelevant to the formal definition in which they had been introduced earlier (see incident 5 and incident 6).

Even further the representations in which students were experienced, and especially the local straightness, did not have for the students the meaning I, as a teacher (and researcher), intended to offer them. This 
meaning of local straightness evolved throughout the episode in parallel with - and in support of - the evolution of students' meaning of the definition of tangent line. In the first three incidents, we had no clear evidence that the magnified image had a special meaning for the students, especially in relation to the existence, or not, of tangent line. The magnified image had the potential to act as a supporting inscription on the screen but, in our case, this was not apparent in the students' arguments. That was rather frustrating for me as most of the experiment structure was based on the idea of local straightness. However, later on, when the example of a non-differentiable function was discussed (Incident 4), in which the local straightness was absent, a kind of connection had began to emerge between the graph's inscription in the magnified image and the existence, or not, of the tangent line. After that, in the next three incidents, the role of the local straightness as mediator in the students' construction of meaning of tangent line was evident. Local straightness supported: the student conflict with regard to tangency at an inflection point (incident 5); the extension of the definition in cases of tangent vertical to $x$-axis (incident 6); and, students' meaning shift from geometrical to analytic perspectives (incident 7).

The role of the examples was crucial in the above process, not only the pre-prepared examples but also the examples that I proposed spontaneously in response to student contributions to the discussion. These examples had several roles in supporting students' construction of meaning. One of these roles was to unsettle the validity of the definitions proposed by the students and to create conflict between their meaning of the definition and the formal meaning. Another role was the establishment of a meaning of local straightness that can mediate students' thinking about tangents. There were cases in which students' unexpected responses caused changes in the pre-planned order in which the examples were introduced. These changes, in the examples and in the order in which they were used, introduced another aspect to the concept of instructional example space proposed by Zazkis and Leikin (2008). This aspect concerns the dynamic nature of the instructional example spaces, when these are applied in real classroom conditions. In this study the instructional example space was not only a didactical tool but also one of the foci of the study. As a didactical tool, this space was based on our knowledge of students' misunderstandings about tangent line and aimed to reveal these misunderstandings and make students rethink about what they are expected to know already. However, these examples, and their sequence, changed in the course of the teaching experiment. Thus, I would say that the dynamic aspect of the instructional example space implies that the set of example needs not only to be well prepared and rich but also to be flexible enough to adjust to unpredictable students' responses.

Students' participation in the classroom discussion offered the opportunity for their personal meanings to be exposed. The polyphony in students' voices, the expression of different perspectives and the fact that there were no shared meanings between students and myself became evident through this discussion. This scientific debate (Bartolini Bussi 1996) was introduced and orchestrated by myself towards a shared understanding. The evolution of students' meanings, revealed through this discussion, was not linear and many conflicts and fluctuations were observed. My role as teacher was to mediate an articulation process 
towards the construction of a shared meaning that is compatible with the formal mathematical meaning (ibid). This role was not straightforward, especially when I realised that my apparently well structured lesson plan did not work exactly as I had intended. As a result, my role fluctuated between orchestrating the discussion and introducing new examples and changing the order of the examples. These examples that constituted the instructional example space might not be included in students' personal example spaces. There were some indications regarding students' example spaces through their participation in the discussion. For example, when I asked the students to offer the definition of tangent line, they proposed their own personal definitions that were different from the definition they had been introduced to earlier in the session. Future research in teaching experiments could investigate how students' example spaces develop in comparison with the instructional example space.

Concluding I would say that there was evidence of evolution in the students' meaning towards a more sophisticated understanding of tangency (second conjecture). This evolution, as I mentioned before, was not linear and was interrelated with the evolution of the other characteristics of the experiment (third conjecture). Thus students' meanings of the definition of tangent line evolved while their meaning of the inscriptions of the electronic environment, the instructional example space, the discussion and the role of the teacher evolved.

Taking into account the above multi-layered set of issues, I would like to put forward the claim that teaching practice informed by research, supported by technology and using flexible instructional example spaces coupled with cautious use of mathematical symbolisation can enhance the students' learning experience. In this approach, the role of the teacher was key to this enhancement, in particular with regard to the capacity to: understand the classroom situation, take decisions and possibly modify the initial plan in the moment, select the appropriate examples and orchestrate the discussion in order to allow the emergence of students' insights and shifts in their personal perspectives.

\section{Acknowledgements}

I would like to thank the students who participated in this study and their teacher, the anonymous reviewers for their useful feedback, Theodossios Zachariades and Despoina Potari for their support throughout the study and Lulu Healy, Victor Giraldo and the Research in Mathematics Education Group at the University of East Anglia, particularly Elena Nardi, for their helpful comments on drafts of this paper.

\section{References}

Artigue, M., Batanero, C., \& Kent, P. (2007). Mathematics Thinking and Learning at Post-secondary Level. In F. K. Lester (Ed.), The Second Handbook of Research on Mathematics Teaching and Learning (pp. 1011-1049). USA: NCTM. Bartolini-Bussi, M. G. (1996). Mathematical discussion and perspective drawing in primary school. Educational Studies in Mathematics, 31(1-2), 11-41.

Bell, A. (1993). Some experiments in diagnostic teaching. Educational Studies in Mathematics, 24(1), 115-137.

Berger, M. (2004). The functional use of a mathematical sign. Educational Studies in Mathematics, 55(1-3), 81 - 102. 
Biza, I. (2008). [Students' intuitive perspectives on the tangent line in the context of Analysis]. Unpublished PhD in Mathematics Education, University of Athens, Athens, Greece.

Biza, I. (2010). Evolution of mathematical discourse with the mediation of electronic environment: The case of tangent line. Paper presented at the Proceedings of the 13th Special Interest Group of the Mathematical Association of America (SIGMAA) Conference on Research in Undergraduate Mathematics Education (RUME). from http://sigmaa.maa.org/rume/crume2010/Archive/Biza.pdf

Biza, I., Christou, C., \& Zachariades, T. (2008). Student perspectives on the relationship between a curve and its tangent in the transition from Euclidean Geometry to Analysis. Research in Mathematics Education, 10(1), 53-70.

Biza, I., Diakoumopoulos, D., \& Souyoul, A. (2007). Teaching analysis in dynamic geometry environments. In D. PittaPantazi \& G. Philippou (Eds.), Proceedings of the 5th Conference of European Research in Mathematics Education (pp. 13591368). Larnaca, Cyprus. Available at: http://ermeweb.free.fr/CERME5b/WG9.pdf.

Biza, I., \& Zachariades, T. (2010). First year mathematics undergraduates' settled images of tangent line. The Journal of Mathematical Behavior, 29(4), 218-229.

Castela, C. (1995). Apprendre avec et contre ses connaissances antérieures: Un example concret, celui de la tangente. Recherches en Didactiques des mathématiques, 15(1), 7-47.

Clandinin, D. J., \& Conelly, F. M. (2000). Chapter 7: Composing Field Texts. Narrative Inquire in Story in Qualitative Research (pp. 92-118). San Francisco, CA: Jossey-Bass.

EucliDraw. (2009). Retrieved 3 October, 2009, from http://www.euclidraw.com

Habre, S., \& Abboud, M. (2006). Students' conceptual understanding of a function and its derivative in an experimental calculus course. The Journal of Mathematical Behavior, 25(1), 57-72.

Harel, G., Selden, A., \& Selden, J. (2006). Advanced Mathematical Thinking. Some PME Perspectives. In A. Gutierrez \& P. Boero (Eds.), Handbook of Research on the Psychology of Mathematics Education: Past, Present and Future (pp. 147-172). Rotterdam, The Netherlands: Sense Publishers.

Kaput, J. (1994). Democratizing acess to calculus: New roots to old roots. In A. Schoenfeld (Ed.), Mathematical Thinking and Problem Solving (pp. 77-156). Hillsdale, NJ: Lawrence Erlbaum.

Lagrange, J. B. (1999). Complex calculators in the classroom: theoretical and practical reflections on teaching pre-calculus. International Journal of Computers for Mathematical Learning, 4(1), 51-81.

Lakatos, I. (1976). Proofs and refutations: The logic of mathematical discovery. Cambridge, UK: Cambridge University Press. Mariotti, M. A. (2001). Justifying and Proving in the Cabri Environment. International Journal of Computers for Mathematical Learning, 6(3), 257-281.

Maschietto, M. (2008). Graphic Calculators and Micro-Straightness: Analysis of a Didactic Engineering. International Journal of Computers for Mathematical Learning, 13(3), 207-230.

Noss, R., \& Hoyles, C. (1996). Windows on Mathematical Meanings. Dordrecht, The Netherlands: Kluwer Academic Publishers.

Presmeg, N. C. (2006). Research on visualization in learning and teaching mathematics. In A. Gutierrez \& P. Boero (Eds.), Handbook of Research on the Psychology of Mathematics Education: Past, Present and Future (pp. 205-235). Rotterdam, The Netherlands: Sense Publishers.

Steffe, P., \& Thompson, P. (2000). Teaching experiment methodology: Underlying principles and essential elements. In A. E. Kelley \& R. Lesh (Eds.), Handbook of research design in mathematics and science education (pp. 267-306). Mahwah, NJ: Erlbaum.

Tall, D. O. (1987). Constructing the concept image of a tangent. In J. C. Bergeron, N. Herscovics \& C. Kieran (Eds.), Proceedings of the 11th Conference of the International Group for the Psychology of Mathematics Education (Vol. 3, pp. 6975). Montréal, Canada. 
Tall, D. O. (1989). Concept images, generic organizers, computers and curriculum change. For the Learning of Mathematics, $9(3), 37-42$.

Tall, D. O., Smith, D., \& Piez, C. (2008). Technology and Calculus. In M. K. Heid \& G. W. Blume (Eds.), Research on technology and the teaching and learning of mathematics: Vol. 1. Research synthesis (pp. 207-258). USA: NCTM, Information Age Publishing.

Vinner, S. (1991). The role of definitions in the teaching and learning of Mathematics. In D. Tall (Ed.), Advanced Mathematical Thinking (pp. 65-81). Dordrecht, The Netherlands: Kluwer.

Vygotsky, L. S. (1978). Mind and society: The development of higher mental processes. Cambridge, MA: Harvard University Press.

Watson, A., \& Mason, J. (2005). Mathematics as a constructive activity: Learners generating examples. Mahwah, NJ: Lawrence Erlbaum.

Watson, A., \& Shipman, S. (2008). Using learner generated examples to introduce new concepts. Educational Studies in Mathematics, 69(2), 97-109.

Winicki, G., \& Leikin, R. (2000). On Equivalent and Non-Equivalent Definition: Part 1. For the Learning of Mathematics, 20(1), 17-21.

Zaslavsky, O., \& Shir, K. (2005). Students' conceptions of a mathematical definition. Journal for Research in Mathematics Education, 36(4), 317-346.

Zazkis, R., \& Leikin, R. (2008). Exemplifying definitions: a case of a square. Educational Studies in Mathematics, 69(2), 131148.

Zazkis, R., Liljedahl, P., \& Chernoff, E. (2008). The role of examples in forming and refuting generalizations. ZDM, 40(1), 131-141. 


\section{List of Figures}

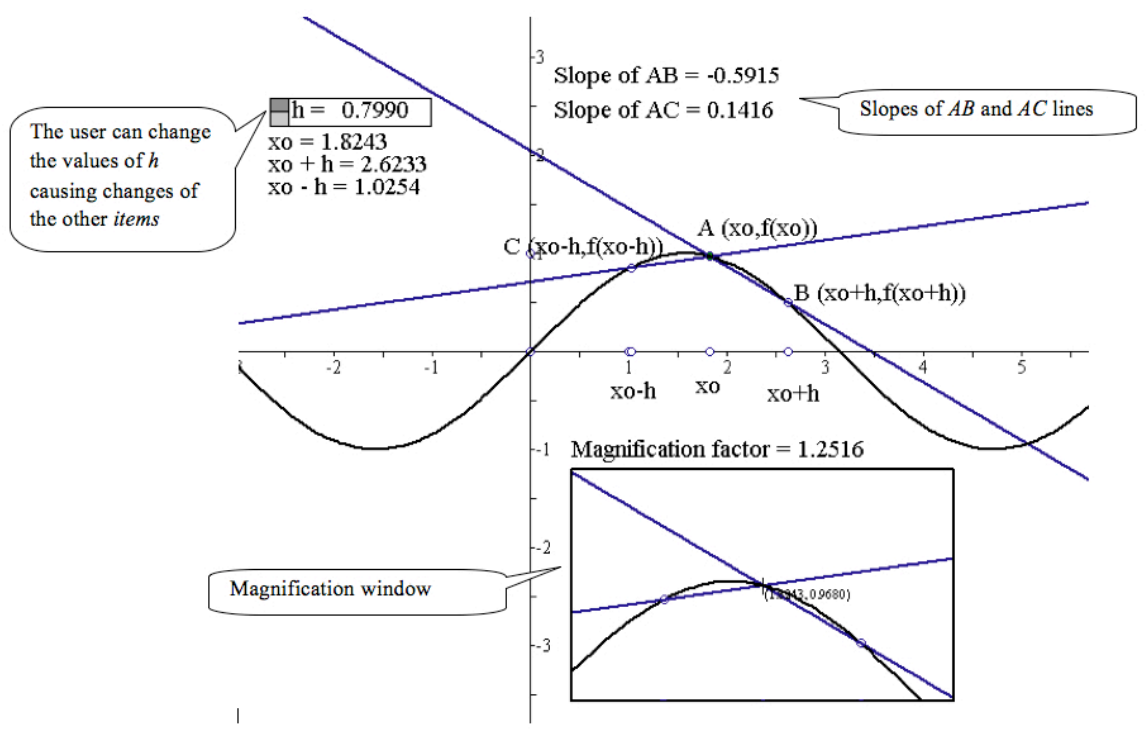

Fig. 1 The electronic environment used in the teaching experiment with the magnification tool (translation from Greek)

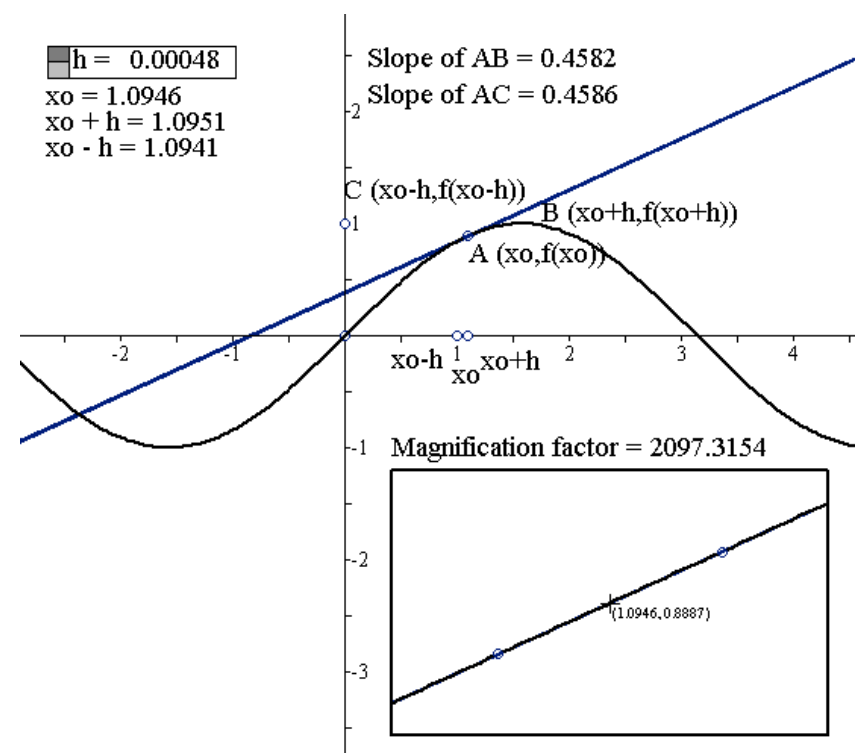

Fig. 2a The tangent line has another common point with the curve except the tangency point

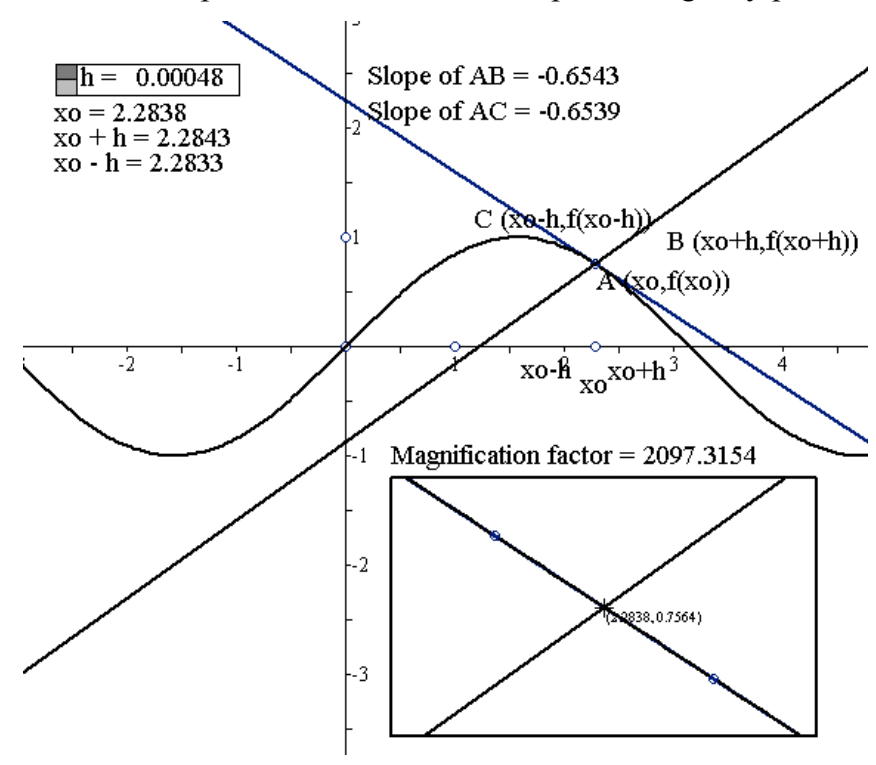


Fig. 2b Only one common point is not a sufficient condition for tangency

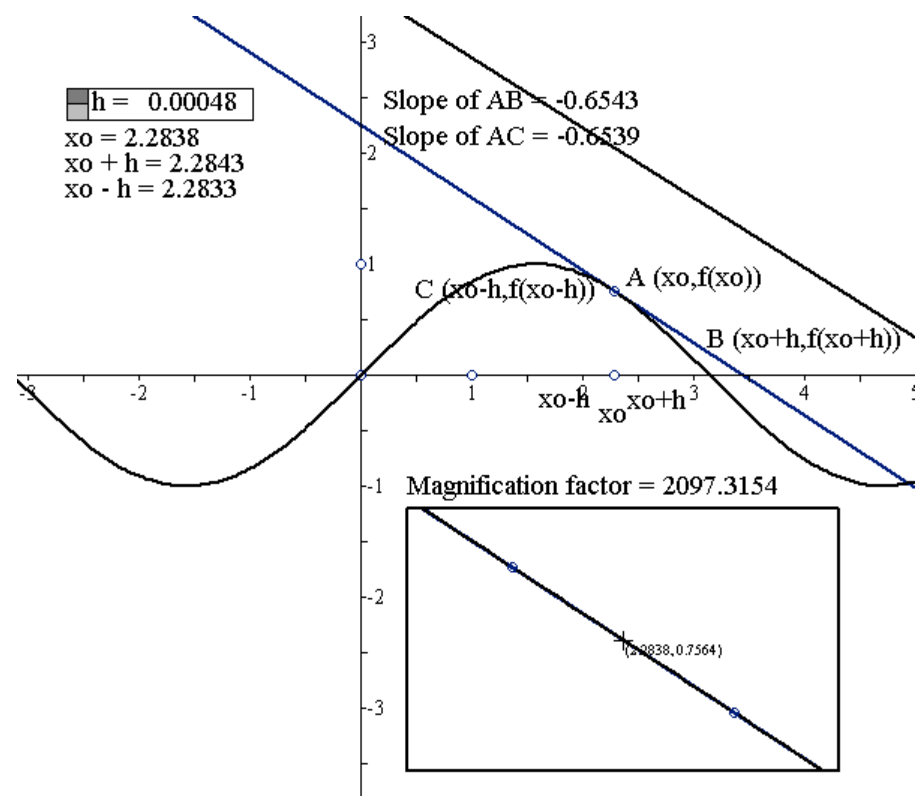

Fig. 2c Equal slopes is not a sufficient condition for tangency

Fig. 2 Examples used for the reconstruction of the definitions 1, 2 and 3

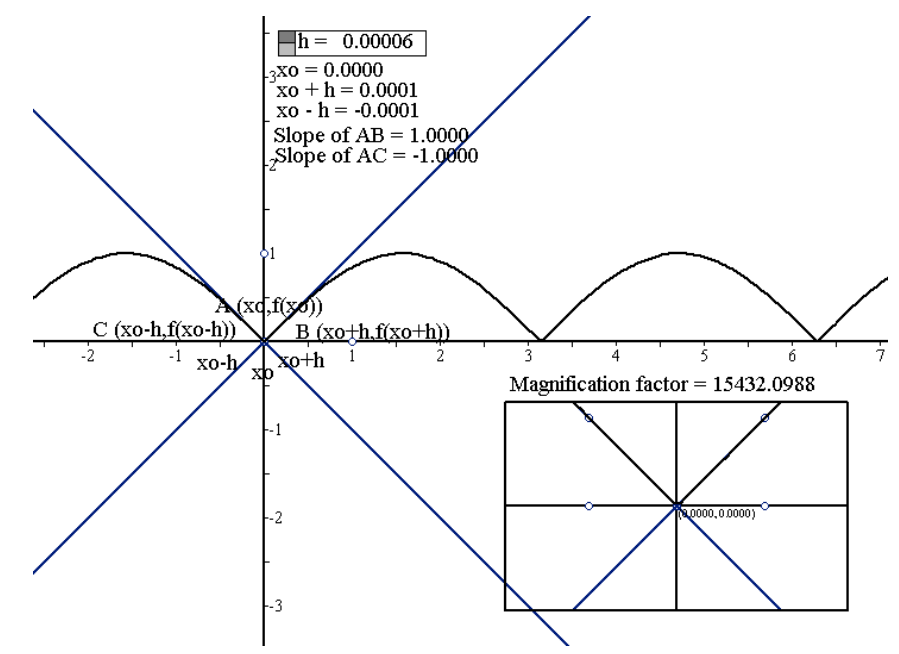

Fig. 3 Non-existence of tangent line at the point $(0,0)$ in which the function is not differentiable

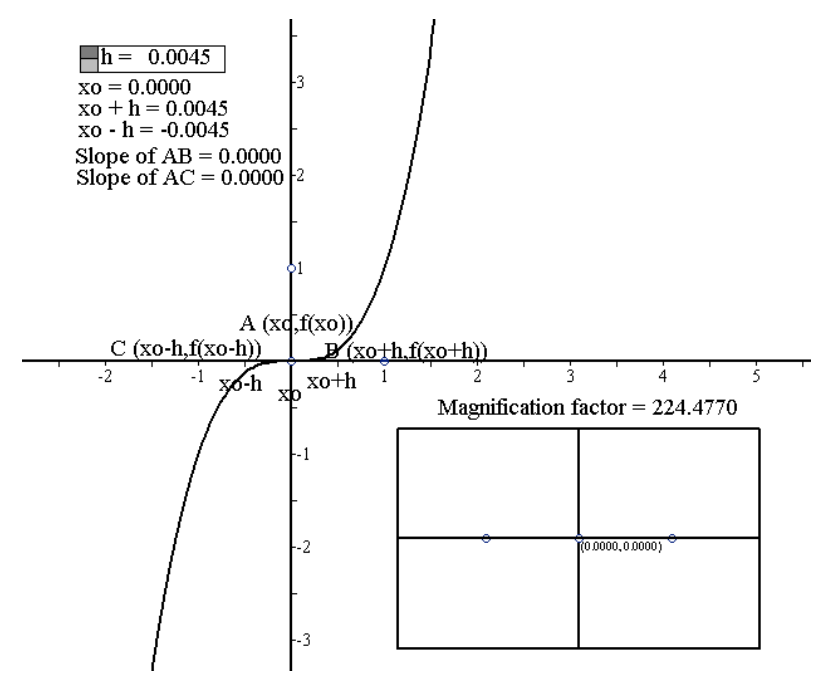

Fig.4 Tangent line at an inflection point 


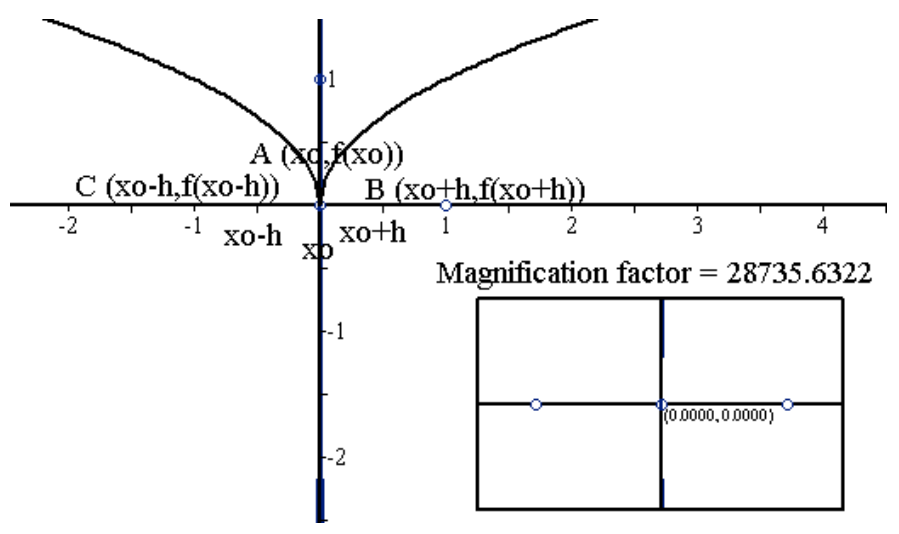

Fig. 5a $A$ is a cusp point

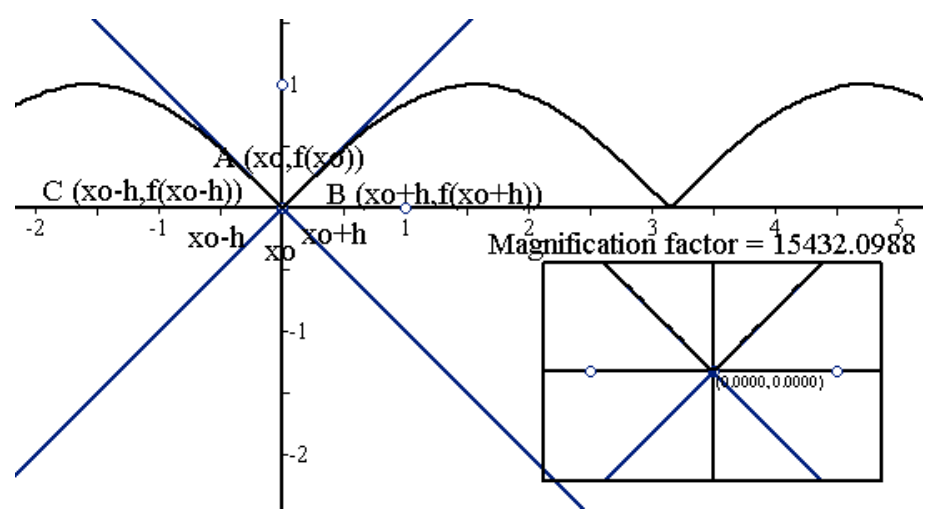

Fig. 5b $A$ is an edge point

Fig. 5 Comparison of the magnified graph at an edge and a cusp point in the electronic environment

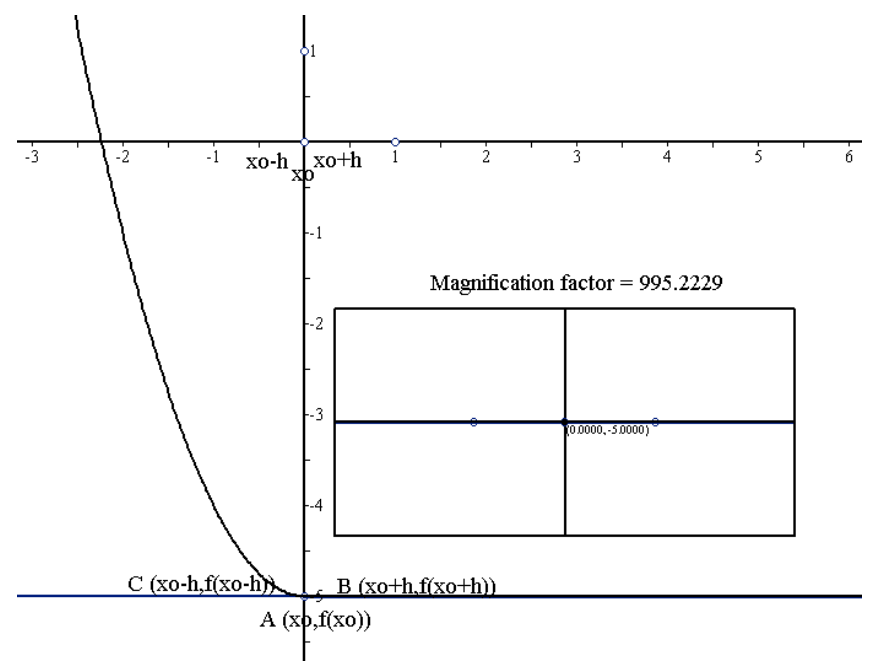

Fig. 6 The tangent coincides with the graph at any region of the tangency point 


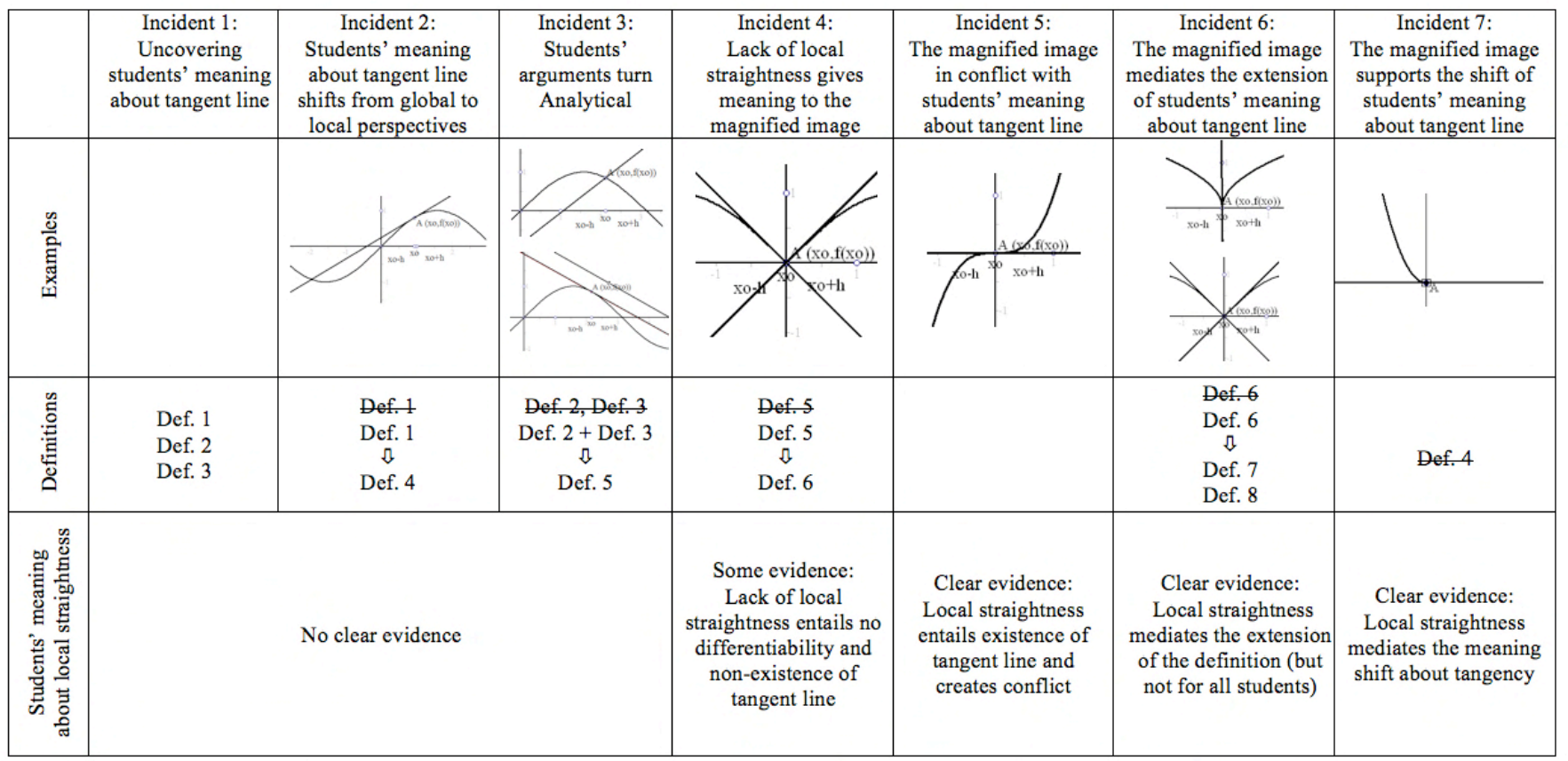

Fig. 7 Evolution of students' meaning about tangent line and local straightness through the instructional example space 


\section{List of Tables}

Table 1 Comparison of results from the pre and post-questionnaire in number of students who gave correct responses

\begin{tabular}{|c|c|c|c|c|c|c|c|c|}
\hline & $I^{(a)}$ & I & $\mathrm{C}^{(\mathrm{b})}$ & I & $\mathrm{C}$ & I & $\mathrm{C}$ & I \\
\hline Pre-questionnaire & 9 & 4 & 6 & 3 & 1 & 9 & 1 & 9 \\
\hline Post-questionnaire & 11 & 11 & 12 & 10 & 8 & 10 & 7 & 10 \\
\hline Difference & +2 & +7 & +6 & +7 & +7 & +1 & +6 & +1 \\
\hline
\end{tabular}

Table 2 The discussed definitions of the tangent line of a function graph

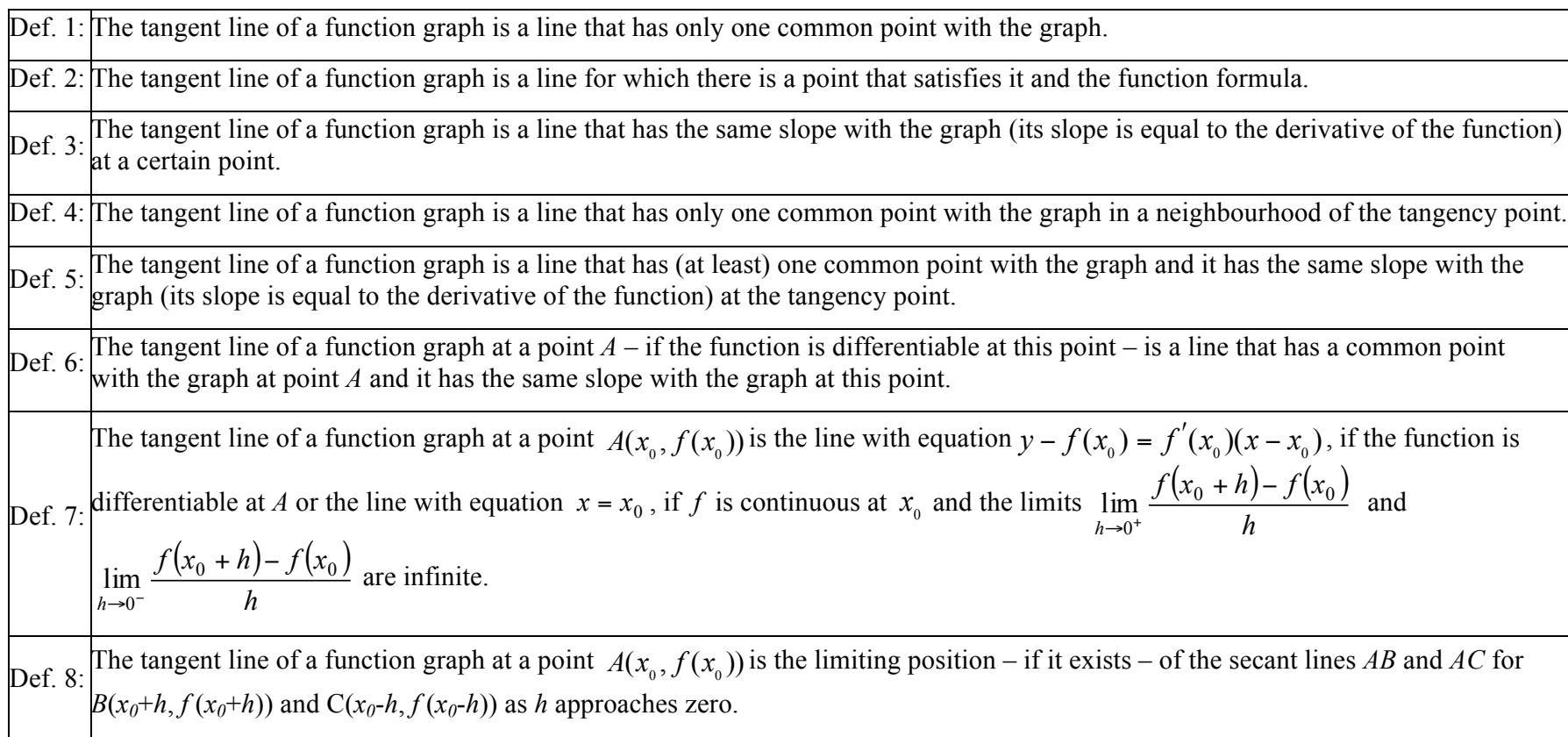

